\title{
Decentralized Global Copyright System Based on Consortium Blockchain with Proof-of-Authority
}

This paper was downloaded from TechRxiv (https://www.techrxiv.org).

\section{LICENSE}

CC BY 4.0

SUBMISSION DATE / POSTED DATE

03-09-2021 / 09-09-2021

\section{CITATION}

Islam, Md. Mainul; IN, Hoh Peter (2021): Decentralized Global Copyright System Based on Consortium Blockchain with Proof-of-Authority. TechRxiv. Preprint. https://doi.org/10.36227/techrxiv.16564218.v1

$\mathrm{DOI}$

10.36227/techrxiv.16564218.v1 


\title{
Decentralized Global Copyright System Based on Consortium Blockchain with Proof-of-Authority
}

\author{
Md. Mainul Islam®, Graduate Student Member, IEEE, Hoh Peter IN॰, Member, IEEE,
}

\begin{abstract}
Conventional copyright systems are governed nationally, and there is no global ledger to store copyright data. Because copyright laws differ across countries, it is difficult to provide cross-border copyright protection. In this paper, we propose a novel decentralized copyright system based on consortium blockchain, which ensures cross-border copyright protection of individuals' digital content and solves existing challenges in international copyright management. The proposed system enables a synchronized platform to register and trade copyright globally without using a global cloud. Individual countries receive membership from a copyright federation and participate in block creation by executing the energy-saving proof-of-authority consensus algorithm. These countries are regarded as the authorities of the platform and are responsible for proposing new blocks after validating transactions. Anyone, either registered or unregistered, can investigate a copyrighted work, but only registered users can make transactions. A token-based payment system is also proposed for paying copyright charges or transaction fees to the authorities through the federation. A prototype of the system was implemented, and its performance was evaluated. This paper provides direction and guidance towards international copyright management.
\end{abstract}

Index Terms-Decentralized copyright, international copyright, cross-border copyright, copyright protection, consortium blockchain, proof-of-authority.

\section{INTRODUCTION}

W ITH the advancement of the Internet and social media, the reproduction of digital content is increasing rapidly worldwide. At the same time, the protection of copyrighted content has become crucial requirement. Content creators must be assured that they can protect their content from piracy while making it available online. Copyright infringement is of great concern worldwide as it causes economic losses to producers, developers, and policymakers. It discourages creative works and hinders the development of science and innovation. Although national copyright systems can protect the copyrighted works of their own citizens, they do not provide sufficient protection for cross-border copyright because they rely on territorial copyright laws [1]. Each country sets its own copyright laws independently. There is no such term as international copyright laws; instead, there are some international treaties on copyright protection [2]. The first acceptable treaty on crossborder copyright protection was the treaty proposed by the Berne Convention of 1886 to set an international agreement for protecting literary and artistic works beyond the borders of individual countries [3]. Today, more than 179 countries worldwide have signed this treaty. The basic principle of the

Md. Mainul Islam and Hoh Peter IN are with the Department of Computer Science, Korea University, South Korea (e-mail: mainul.islam@ieee.org; hoh_in@korea.ac.kr).

Manuscript received MM DD, YYYY; revised MM DD, YYYY. treaty is regarding legitimate rights for the protection of literary and artistic creations that originated in a contracting state. According to the treaty, if a copyright is infringed by someone in a foreign territory and the convention is available, the copyright owner is treated as a citizen of the foreign territory and must be prepared to proceed with the national copyright laws of that territory, which is termed as "national treatment" [2]. The Bern Convention has not defined a specific court for conducting national treatments; instead, the treatments are practiced in the local jurisdictions of the foreign territories where the copyright infringements occurred. The minimum protection period defined by the convention is 50 years after the owner's death for an artistic work or publication and 25 years for a photograph. The minimum protection standards regarding copyrighted works are the rights to reproduce or make copies, distribute, translate, make adaptations, recite or perform publicly, and broadcast. There also exist several global organizations, such as the World Intellectual Property Organization (WIPO), International Federation of Reproduction Rights Organizations (IFRRO), European Commission Copyright International, Copyright Clearance Center (CCC), and RightsDirect, who aim to protect copyright from a global perspective but still cannot achieve complete harmonization in cross-border copyright protection. The differences in national copyright laws, absence of a global copyright monitoring system with a central cloud that can store copyrighted content worldwide, and lack of transparency raise challenges in international copyright management for these organizations [4]. Copyright information is dispersed across various centralized databases, which are not incentivized to share. It is impossible to bring all centralized servers that store billions of copyright data under a synchronized platform. Due to the lack of a global copyright monitoring system through which people can investigate whether a work is copyrighted by searching copyright catalogs and records, copyrighted works are often downloaded from Google or social media unconsciously and shared with others freely, which may result in huge revenue losses to copyright holders. If a copyright infringer is foreign to the copyright's country of origin, it is difficult to take them to a local court for punishment, unless the countries of the copyright owner and copyright infringer have a good judicial relationship. For example, the copyrighted works of American citizens may be protected in another country under certain conditions if that country comes to an international copyright agreement with the United States of America [5]. Protection against unauthorized reproduction of copyrighted works in a particular country depends on the national copyright laws of that country. However, executing punishment for foreign citizens because of copyright infringement according to local 
copyright laws is difficult. Therefore, international copyright management with international copyright laws is urgently needed in this era of globalization. The proper implementation of international copyright laws can mitigate copyright infringement by preventing digital content from being used in ways the owners do not wish and ensuring that the content owners are the only person who have the right to monetize their content. Unauthorized persons cannot make copies of a copyrighted work or exercise the rights they do not have. A copyright owner can take someone to an international court for infringing on their copyright and recover the economic losses caused by the infringement. Penalties and punishments for copyright infringements vary based on the statuses of copyrighted works and the intention of copyright infringers.

To meet the requirements for international copyright management, a blockchain-based global copyright system can play an important role by enabling a decentralized shared ledger in which worldwide copyright data can be recorded in a neutral, distributed, and tamper-proof manner without any manual operation [6]. The data will be timestamped and cryptographically connected in chronological blocks across a synchronized network. Copyrighted content throughout the world can be easily recognized and verified publicly. Blockchain can reduce the need for trusted third parties and establish mutual trust among unknown entities. This meets the necessary requirements, but several questions must be answered, such as how to register digital content for copyright, where to store the copyrighted content, and what type of blockchain should be used?

While reviewing a copyright application in a national copyright system, there is no ability to search or compare the claimed work with other works to justify whether similar works were previously registered in the system's database or elsewhere. A copyright office does not knowingly register a duplicate or ambiguous claim that is available in the public domain. If a registered claim is discovered to be an exact duplicate of another claim, the copyright office may refuse the registration and apply punishment for this crime according to the corresponding national copyright laws [5]. In contrast, blockchain can detect and refuse a duplicate claim immediately at the time of registration if all copyrighted content is available on it. However, the storage of massive files in blockchain is a significant problem due to high memory consumption and network overhead. For example, the size of the Bitcoin blockchain doubles each year although the blockchain contains only metadata about transactions and limits the block size to 1 MB [6]. Since January 2009, the size has been increasing exponentially, reaching almost 354 GB in July 2021 for a total of 690,720 blocks. Therefore, it is unwise to store copyrighted content in a blockchain. Instead, the content can be stored in a separate server, and their locations can be included as metadata in transactions. However, location addressing creates another issue in the case of changing the URL of some content or the server being somehow unavailable. To solve this issue, the interplanetary file system (IPFS), a peer-to-peer (P2P) file sharing protocol, has introduced content addressing in which each content is identified by a unique address called a content identifier (CID) [7]. Each CID is derived from each content using cryptographic hash functions called multihashes. Content is permanently stored in a distributed network and accessed by its CID (https://ipfs.io/ipfs/CID). The non-fungible token (NFT) system, EIP-721, is currently using IPFS for ERC-721 NFT storage and verification [8]. An NFT is a unique digital art that is considered a crypto asset and can be exchanged with cryptocurrencies. This is one possible approach to protect copyrights because it refers to a digital object that cannot be interchanged. However, there are several challenges to NFT-based copyright protection. First, EIP-721 runs on the Ethereum platform, which is a public blockchain network. Therefore, controlling the network by a global community is impossible. Users remain anonymous in this network, which may create opportunities for copyright infringement by criminals. Second, it operates based on smart contracts that deal with the ether cryptocurrency, which limits NFT trading to ether. The final and greatest drawback of this system is that it stores an NFT object in IPFS and records the metadata of the object in the blockchain. IPFS distributes a file in a P2P network, which leaves a contradiction in copyright registration because multiple persons can claim ownership of the same file. An owner can protect file privacy by encryption, but the ownership of an encrypted file cannot be verified without revealing the owner's private key, which is a privacy issue. Encryption can prevent others to read the file, but it fails to provide proof of ownership in such a decentralized platform. Therefore, the NFT concept is not appropriate for copyright protection.

This study aimed to solve the data synchronization and copyright verification issues of international copyright management by designing a consortium blockchain-based crossborder copyright protection model.

\section{A. Related Works}

Several papers on copyright protection exist in the literature, mainly focused on centralized copyright management. Only a few papers provide solutions for cross-border copyright protection using decentralized platforms. The papers [9]-[28] have proposed several watermarking techniques for copyright protection. Watermarking protocols can protect the copyrights of photographic, textual, and visual works only. Hence, their use cases are limited from a global copyright perspective, which should cover all types of digital content. Moreover, most watermarks cannot be easily verified, fail to prove ownership, and are vulnerable in this era of advanced editing technology [29], [30]. In [31], the authors have designed smart contract-based digital license management system for $3 \mathrm{D}$ printing or additive manufacturing. To ease the problems of vulnerability and low traceability in traditional watermarkingbased intellectual property protection, a traceable copyright protection scheme has been proposed in [32], which is resistant to multiple embedding attacks. A quadratic matrix transformation model is adopted for watermarking in the blockchain environment, which transforms copyright information into several encrypted fragments to hide the real watermark positions. However, the function of blockchain in this scheme is missing and questionable. This paper has not clarified 
where copyrighted content would be stored (off-chain or onchain), what type of blockchain would be used, and which consensus mechanism would be operated. In [33], the authors have introduced an efficient and secure approach for digital content trading using a combination of off-chain and on-chain blockchains. User authentication and copyright registration are processed in a centralized platform that includes a server to store encrypted content. To cope with the storage capacity limitation of on-chain blockchain, copyright consumers create some secret blocks that contain encrypted content and transaction details. The headers of the secret blocks are stored in a public blockchain as transactions. Both authenticated and unauthenticated users maintain the public blockchain network by executing a weight-based byzantine fault tolerance (WBFT) consensus algorithm, where authenticated users have higher weights and more chance to be elected as validators. This type of approach is well-suited to small-scale environments where the number of users is limited. However, it cannot meet the requirements of a global platform with billions of users because latency increases and throughput decreases as the number of users increases.

\section{B. Our Contributions}

The key contributions of this study can be summarized as follows:

- A consortium blockchain is adopted for cross-border copyright protection instead of a public blockchain so that member countries under a copyright federation can validate transactions and govern the network with equal rights and mutual trust.

- The energy-saving proof-of-authority (PoA) consensus algorithm is used for block mining, where member countries act as the authorities. This does not require high computing power since there is no cryptographic puzzle solving as there is in proof-of-work (PoW).

- Unlike IPFS, elliptic curve cryptography is used for content addressing so that the real owners can prove their content creations. Miners can validate ownership by verifying transaction signatures.

- Copyright owners are free to keep their content anywhere, even on their local storage devices. Verifiable public keys linked to the content are recorded in the blockchain as metadata, which is a safe method of copyright registration. This does not require a centralized server to store copyrighted content globally; therefore, there is no encryption-decryption process. Copyrighted content can be published or advertised on digital marketplaces once it has been registered on the blockchain.

- Unlike the ERC-721 NFT standard, the proposed system supports off-chain ownership transfer with fiat currencies, where a seller delivers the private key of an auxiliary public key regarding a copyrighted work to a buyer as proof of the ownership transfer. The buyer can record the purchase in the blockchain by making an on-chain transaction with sufficient proofs. To pay the transaction fee, a token must be collected from the federation and included in the transaction. Transaction fees are distributed among miners according to their mining services.
- A prototype of the system was implemented on an Oracle VM VirtualBox to evaluate the system's feasibility, and its performance was analyzed based on the computing power and network connectivity of the miners.

The remainder of this paper is organized as follows. A background of this research is given in Section II. The proposed system and algorithms are presented in Section III. The security of the system is analyzed in Section IV. The implementation results and performance analyses are presented in Section V. Finally, the paper is concluded in Section VI.

\section{BACKGROUND}

\section{A. Copyright}

Copyright is an intellectual property that protects its owner's original work of authorship as soon as the work is fixed in a tangible medium of expression [34]. The work can be any type of digital content, including articles, books, newsletters, emails, images, sound recordings, videos, architectural designs, software, and computer programs. The term "original work of authorship" refers to a work that is created by an author independently with a minimal degree of creativity, excluding its title, name, symbols, short phrases, and colors. Copyright does not protect the concept, idea, principle, and method of content creation; rather, it protects the way in which these things are expressed [34]. It preserves the format of the content but not the information of the content. Minor variations in datasets may be sufficient to abolish copyright protection. The protection applies from the moment of putting an original work of authorship in a tangible form under a sufficiently permanent or stable organization such that the work can be reproduced, perceived, and publicly accessed for more than a transitory time period [5].

The objective of copyright is to encourage the development of science and innovation by supporting content creators with financial benefits for their creative works. Copyright provides a content creator with the rights to reproduce their content via copies, distribute the copies, license the copyright, create adaptations, translate the content, broadcast the content through media, display the content publicly, and reuse the copyrighted materials in another work. This generally covers a single original work of authorship that belongs to one or multiple author(s) who create(s) the work of expression. When multiple authors materially contribute to a single work with the intention of merging their contributions into inseparable parts of a unitary whole, the work is referred to as joint work (e.g., a book or article), and the authors are considered as joint authors [5]. In the case of a joint work, each author owns the work equally, earns equal profit, and can exercise all of the rights inherent to the work by default, unless they sign a written agreement of rights management and revenue sharing that states otherwise. Any joint author can transfer their ownership whole or in part to another person without the consent of others. If a copyright owner transfers all of the rights associated with a copyrighted work to a person without any condition, it is termed as an assignment. When the owner transfers some specific rights to someone, it is 
known as a license, which can be either exclusive or nonexclusive. An exclusive license allows only the licensee to exercise the rights associated with the license, while a nonexclusive license allows the licensor to license the same rights to others [34].

\section{B. Consortium Blockchain}

A federated or consortium blockchain is a semi-public blockchain that comprises the features of both public and private blockchains [35]. It is also a permissioned blockchain in which a group of miners governs the platform and manages the blockchain. Each miner refers to a single organization or authority in the network. However, the architecture of a consortium network and the rights of the participant nodes depend on the requirement and vary from platform to platform. For a limited number of miners, a consortium blockchain could be the best choice due to its following advantages:

- Low Energy Consumption: Because a limited number of nodes participate in consensus, the energy-intensive PoW algorithm can be avoided. Alternatively, eco-friendlier consensus algorithms can be used [35].

- High-Speed Consensus: Because there are few miners, a consensus can be reached quickly with less effort.

- High Throughput: A faster consensus algorithm increases the number of transactions processed per second.

- No Risk of 51\% Attack: This is the most attractive feature of consortium blockchains. Although blockchain offers a greater level of security, a $51 \%$ attack is a crucial threat to a permissionless platform [35]. If $51 \%$ of all miners on the platform are from a single organization, they can override or reverse a transaction by taking up more than half of the computational power of the system. They can also modify a block by creating a new branch of blocks that would be the longest blockchain in the network. However, a $51 \%$ attack is impossible in a consortium blockchain because the miners of such a network are from multiple organizations and must join through proper authorization.

- No Criminal Activity: A miner cannot be anonymous in a consortium blockchain; therefore, it is impossible to perform malicious activities.

- Regulation: In a consortium blockchain, all nodes must obey the rules of the network. When everyone in the network knows each other, the chance of illegal activities becomes very low. This ensures proper regulation in an enterprise environment.

Consortium blockchains would be most beneficial for an infrastructure in which multiple organizations require a common ground to conduct transactions or share information. For this reason, we adopt a consortium blockchain for our proposed global copyright system, where people throughout the world can read the blockchain, but only authorized miners can write it.

\section{Proof-of-Authority (PoA)}

PoA is a reputation-based consensus algorithm that provides an efficient solution for private and consortium blockchains.

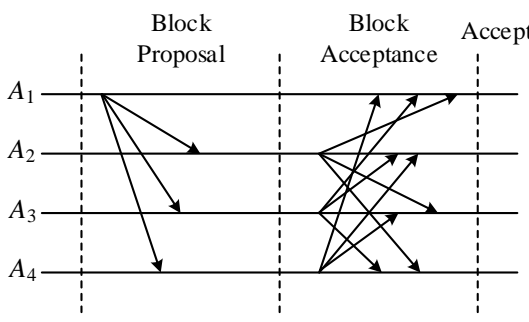

(a)

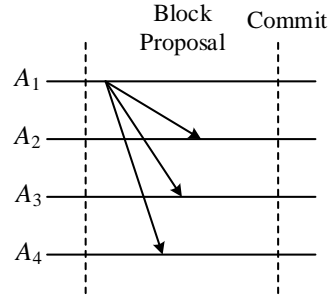

(b)
Fig. 1. Message patterns of PoA schemes: (a) Aura and (b) Clique [36].

Unlike PoW, it does not include the longest chain or confirmation rule. New blocks are directly accepted and added to the chain with the unanimous approval of a limited number of trusted miners. There is no competition for cryptographic puzzle solving among miners. Hence, low computing power is required to execute this consensus mechanism. As the name suggests, PoA depends on a set of $N$ trusted nodes called the authorities, each of which is identified by a unique ID or public key. To ensure transparency in the network, a majority (at least $N / 2+1$ ) of authorities must be honest, and as such, all authorities closely monitor the actions of each other. Consensus in PoA is achieved through a round-robin schedule, where the responsibility of block creation is assigned to each authority in turn.

PoA has two different implementations, called Aura and Clique, which were originally proposed as part of the Ethereum blockchain for private networks [36]. The message patterns of the Aura scheme are shown in Fig. 1(a). The first round covers the block proposal by the current leader, and the second round is required to obtain block acceptance from the non-leader authorities. The block is added to the blockchain after receiving acceptance from the majority of authorities. If the current leader broadcasts a malicious block, the block is discarded, and the leader is kicked out by the honest majority of the network through a voting process. Fig. 1(b) illustrates the message patterns of the Clique scheme, where there is no block acceptance round. While Aura is based on time scheduling, Clique works on the current step and related leader authority following a sequence that considers the block number and number of authorities. At each step, $N / 2-1$ authorities, including the leader authority, can propose blocks. Hence, each authority is allowed to propose a block after every $N / 2+1$ steps. Blocks proposed by the leader authority usually win because each non-leader authority is paused from proposing a block for a random time so that the block from the leader authority reaches everyone first. Because more than one authority can propose a block at each step, forks can occur. A fork is solved by accepting one of the blocks proposed at each step using the GHOST protocol [37] or block scoring approach with the leader's block assigned a higher score. Like Aura, if an authority broadcasts an invalid block, other honest authorities can cast votes to ban the authority from further proposing any block.

Although both PoA approaches have their own advantages and disadvantages, we adopt the Aura approach for our system 

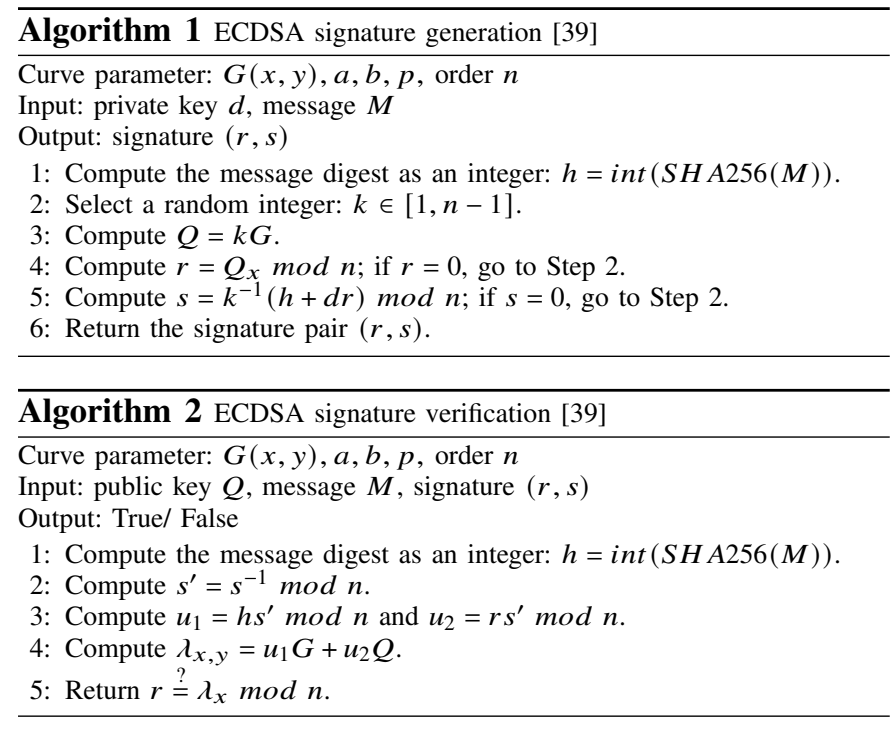

to avoid forks and achieve better synchronization with higher security in the consortium network.

\section{Cryptographic Primitives}

Cryptographic hash functions and digital signature schemes are two indispensable aspects of blockchain. A hash function is a collision-resistant one-way function used to map data of arbitrary size to an irreversible string of fixed length. It converts data into a unique digest, which is considered a digital fingerprint of the data. Two digests cannot be identical unless the input datasets are identical. Minor variations in a dataset lead to different hash values. Several hash functions that are frequently used in cryptocurrencies include SHA256, Keccak256, Keccak512, blake256, and RIPEMD160 [38]. The hash functions Keccak256, Keccak512, blake256, and RIPEMD160 are used for pseudonymous crypto address generation. Because the proposed system adopts a consortium blockchain in which participants are not anonymous and mutual authentication among authorities is required, cryptographic public keys are used as participant IDs instead of pseudonymous crypto addresses. Only the SHA256 hash function is used for content addressing and digital signature generation.

The elliptic curve digital signature algorithm (ECDSA) is adopted for transaction signing and verification. ECDSA is the most popular digital signature scheme for blockchain. It works on some general elliptic curves, such as NIST P-256, secp256k1, and secp256r1.

An elliptic curve over a prime field $\mathbb{F}_{p}$ is given by the equation [39]:

$$
E_{a, b}: y^{2}=x^{3}+a x+b
$$

where $p$ is a prime number and $x, y, a, b \in[1, p-1]$ with

$$
4 a^{3}+27 b^{2} \neq 0
$$

Secp 256k1 is a version of $E_{a, b}$, which is widely used in blockchain. For this curve $a=0, b=7$, and $p=2^{256}-2^{32}-977$ [40]. Therefore, the curve can be expressed as follows:

$$
E: y^{2}=x^{3}+7
$$

A public key $Q$ is a point on $E$ computed by multiplying a base point $G$ with a scalar or private key $k$ such that $Q=k G$. This multiplication is called elliptic curve point multiplication (ECPM), which is the key operation of elliptic curve cryptography and the most time-consuming operation in ECDSA [41]. The ECDSA signature generation and verification mechanisms are shown in Algorithms 1 and 2, respectively [39]. The signing process takes a private key and a message as inputs and generates a two-part signature as output. The inputs of the verification process are a public key, message, and corresponding signature of the message.

\section{System DeSIGN}

The proposed global copyright system is illustrated in Fig. 2 , in which a copyright federation provides membership to individual countries and tokens to users. Member countries are referred to as authorities who maintain a consortium blockchain by executing the Aura PoA consensus mechanism. Each authority has two separate local queues: a transaction queue $q_{T}$ (mempool) and a pending block queue $q_{B}$. The authorities are assumed to be synchronous within one epoch time $t_{e}$, where the creation of the first or genesis block is considered as an epoch. For a certain time, $t_{e}$ is calculated as follows:

$$
t_{e}=t-t_{0}
$$

where $t$ is the Unix timestamp of that particular time, and $t_{0}$ is the Unix timestamp of the genesis block.

If $N$ is the number of authorities in the network and $\delta$ is the duration of each step, the step index $s$ is determined as $s=t_{e} / \delta$, and the current leader is identified as $A_{i}$, where $i=s \bmod N$. The step duration depends on $N$, the block volume, and average computing power of the authorities. If $\lambda$ is the number of transactions per block and $\mu$ is the average processing speed of the authorities, $\delta \propto \lambda N / \mu$. The value of $\delta$ is chosen such that no authority lags behind the tasks of transaction validation, block creation, and receiving acceptances because of slow speed or poor network connectivity. In addition, minimum requirements of processor configuration and network connectivity can be set for the authorities. The current leader at step $s$ proposes a block including valid transactions from the mempool and sends it to the nonleader authorities. If no transaction is available at this step, an empty block is sent. After receiving and validating the proposed block, each authority stores it on $q_{B}$ and sends the acceptance feedback to all other authorities. As soon as the block receives $N / 2+1$ acceptances, it is moved from $q_{B}$ to the main blockchain, and the common transactions between the block and $q_{T}$ are deleted from $q_{T}$.

Someone who wants to make a transaction of copyright registration or trading must be registered in the network by their local government. However, copyright verification does not require any registration. Anyone can verify the copyright of digital content by simply uploading the content or submitting its CID to a global website operated by the federation. When digital content is uploaded to the website, the website computes the CID of the content and shows its copyright information (if available) after interacting with the blockchain. 


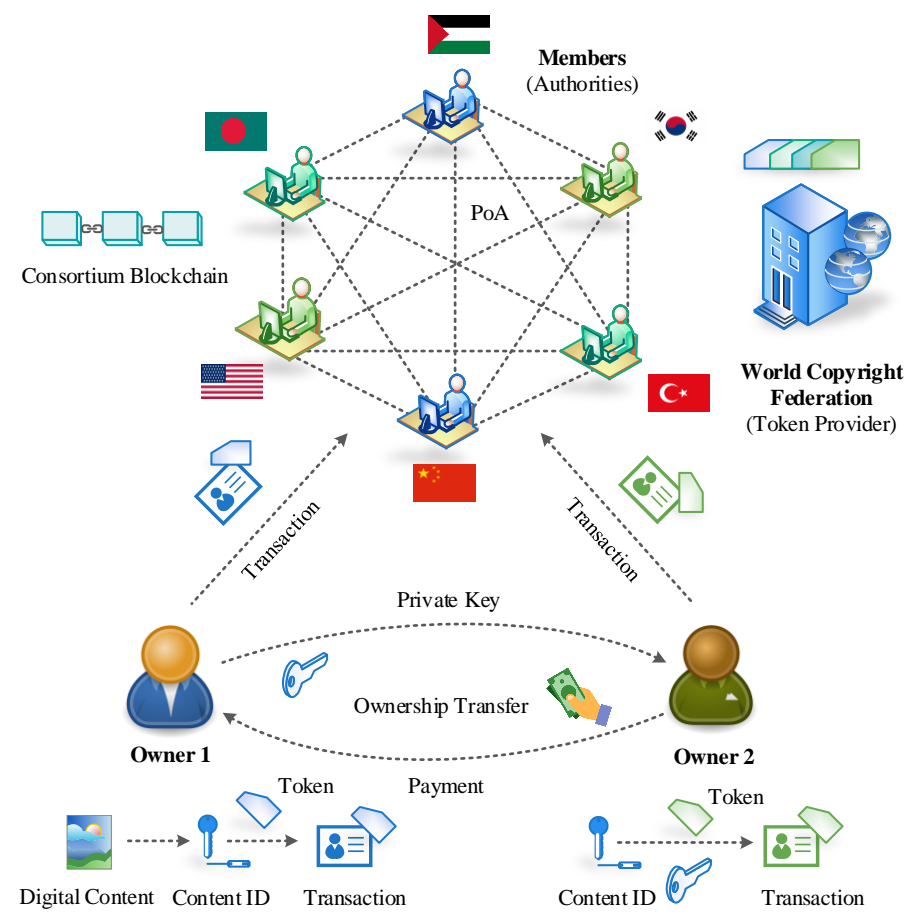

Fig. 2. Overview of proposed global copyright system.

Algorithm 3 demonstrates the steps of copyright registration in the consortium network. To register a copyright, a user first reads a file and converts the file content $C$ (hex value) to a 256-bit private key integer $k_{c}$ using the SHA256 hash function. A public key $Q_{c}$ is generated by performing ECPM with $k_{c}$, which is the CID of the file. A secondary private key $k_{s}$ and the corresponding public key $Q_{s}$ are computed by combining the primary (wallet) private key $k_{p}$ and $k_{c}$. The secondary public key is a proof of the content creation by the user as it is linked to the user's wallet. A tertiary private key $k_{t_{1}}$ and corresponding public key $Q_{t_{1}}$ are created so that the owner can transfer ownership in the future. Because the content is not registered previously, the reference $R$ is zero. A transaction $T$ is formed including $R, Q_{p}, Q_{s}, Q_{t_{1}}, t$ (a timestamp), and $\tau$ (a token). $T$ is signed with $k_{s}$, and the signature $S$ is inserted into the transaction. Finally, the transaction is broadcast to the consortium network, validated by the authorities, and recorded in the blockchain. In the case of joint authorship, the same CID is shared among all joint authors of a work. For $n$ joint authors, $n$ primary keys, $n$ secondary keys, $n$ tertiary keys, $n$ signatures, and an agreement (optional) are included in a registering transaction. If the authors provide a mutual agreement, all rights and revenues are distributed among them according to the agreement; otherwise, they own the content equally and can exercise equal rights by default. Each joint author can transfer their ownership to a person without the consent of the other joint authors by delivering their own tertiary private key.

For selling the copyright of registered content, a seller delivers the CID $Q_{c}$, transaction hash $T_{h}$, and tertiary private key $k_{t_{1}}$ of the content to a buyer. $T_{h}$ is collected from the blockchain in which the transaction regarding $Q_{c}$ is stored.

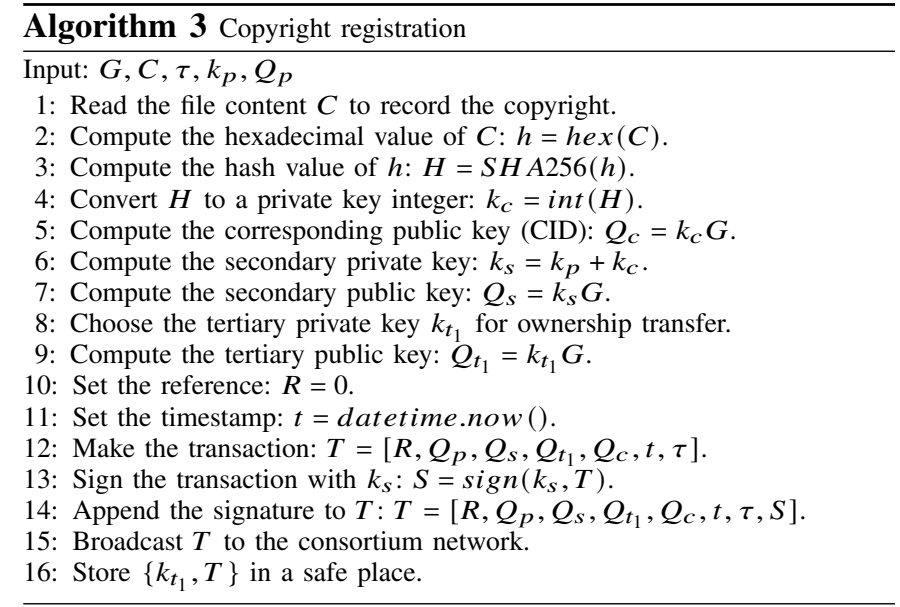

As shown in Algorithm 4, the buyer first checks whether $T_{h}$ is a valid transaction hash or not. The buyer also confirms that the seller is the last owner of $Q_{c}$ by ensuring $T_{h}$ as the last transaction hash regarding $Q_{c}$. If everything is satisfied, the buyer makes a transaction in a similar manner to Algorithm 3 , except the reference $R$ and secondary private key $k_{s}$. In this case, $R$ is set to $T_{h}$, and $k_{s}$ is computed by adding $k_{t_{1}}$ to the buyer's primary private key $k_{p}$, which is proof of the ownership transfer. Fig. 3 illustrates the transaction chain of ownership transfer to the successive owners of a digital item. Each owner holds three public keys for the item, which are referred to as primary, secondary, and tertiary keys. The primary public key is the wallet address of the owner, the secondary public key is the proof of content creation or purchase, and the tertiary public key is required for ownership transfer. A token is required to pay the transaction fee set 


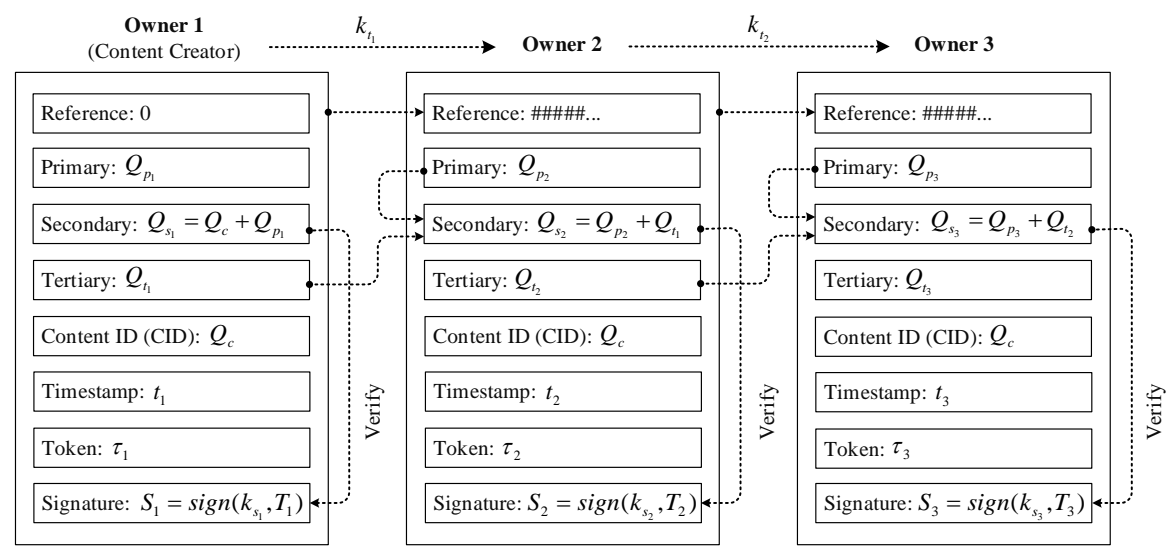

Fig. 3. Transaction chain of copyright registration and trading.

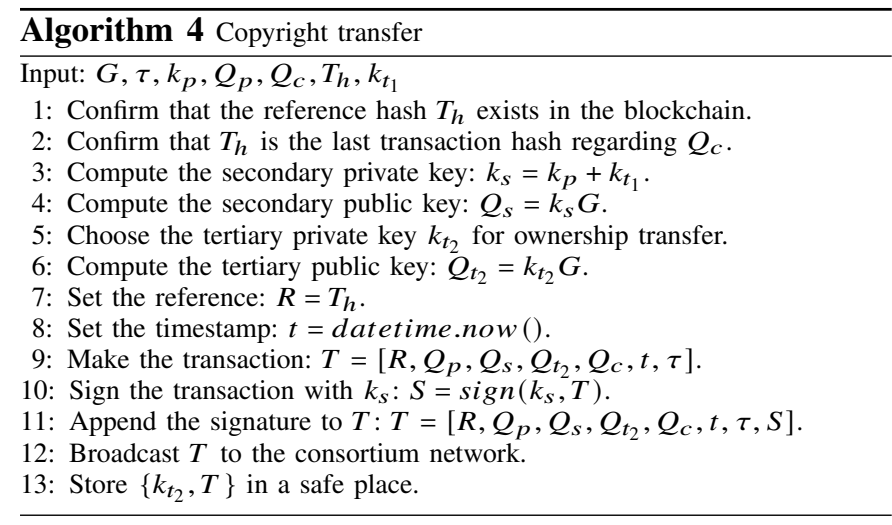

by the network, which can be collected from the federation. Because the proof of copyright purchase is verified by the secondary public key of the current owner, the key is generated by adding the primary public key of the current owner to the tertiary public key of the previous owner from whom the copyright is transferred. In this manner, a chain of ownership transfers is established. To protect a transaction from being tampered, it is signed with the corresponding private key of the secondary public key that the transaction contains. Thus, the signature can be verified by the secondary public key, which proves that the sender of the transaction really has the secondary private key with which the transaction is signed.

Fig. 4 illustrates the proposed token collection scheme, where a user collects a token from the copyright federation in several consecutive steps. The user first sends their wallet public key $Q_{p}$ to the federation. The federation checks whether the key is registered or not. If the key is found to be registered, a token code $\tau_{c}$ is sent to the user. The user signs the code using the wallet private key $k_{p}$ and delivers the signature $S_{p}$ with a token fee to the federation. The federation verifies $S_{p}$ using $Q_{p}$, signs it using the private key $k_{F}$, and delivers the signature to the user. Upon receiving $S_{F}$ from the federation, the user finally makes a token including $\tau_{c}, S_{p}$, and $S_{F}$.

The transaction validation mechanism is described by Algorithm 5 , where an authority takes a transaction $T$ and the public key of the federation $Q_{F}$ as inputs. First, the authority inquiries whether the primary public key $T[1]$ is registered and

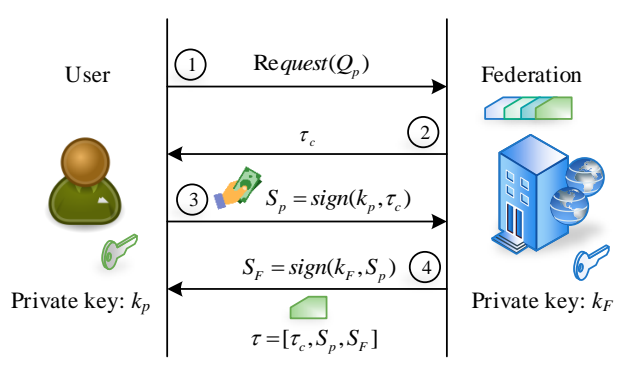

Fig. 4. Proposed token collection scheme.

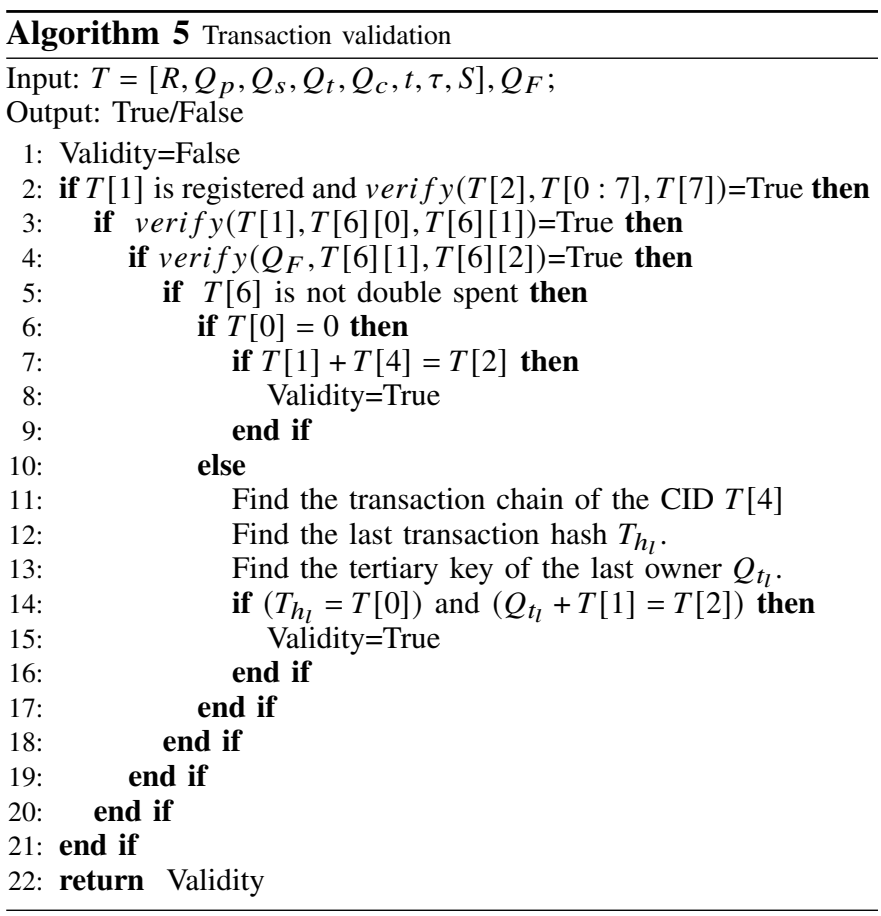

the transaction signature $T$ [7] is valid or not. If the transaction requester is found to be a valid user of the system and $T$ [7] is verified with the secondary public key $T$ [2], the validation process is continued. Second, the authority validates the token $T[6]$ by verifying the signatures $T[6][1]$ and $T[6][2]$ using $T[1]$ and $Q_{F}$, respectively. If the token is found to be valid and not spent previously, the authority checks whether the 


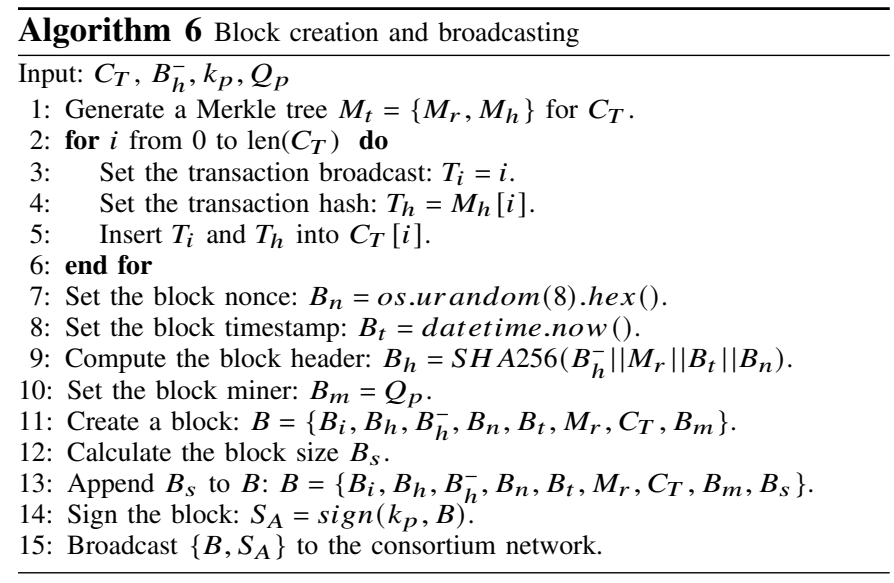

reference $T[0]$ is set or not. If $T[0]$ is null, $T$ is assumed to be a copyright registration request. For a valid transaction, the point addition of $T[1]$ and the CID $T$ [4] must be equal to $T$ [2]. If $T$ [0] is set, $T$ is considered as a copyright transfer request. In such case, the owner of the referenced transaction must be the last owner of the content whose ownership is to be transferred, and the point addition of the tertiary public key of the last owner $Q_{t_{l}}$ and $T$ [1] must be equal to $T$ [2]. If all validation criteria are satisfied, the transaction is confirmed to be stored in a block.

Algorithm 6 demonstrates how an authority creates and broadcasts a block with confirmed transactions. A number of confirmed transactions $C_{T}$, the previous block header $B_{h}^{-}$, and the private-public key pair $\left(k_{p}, Q_{p}\right)$ of the authority are taken as inputs. First, a Merkle tree $M_{t}$ is generated for $C_{T}$, where $M_{r}$ and $M_{h}$ denote the Merkle root and hash, respectively. Each transaction of $C_{T}$ is identified by a transaction index $T_{i}$ and transaction hash $T_{h}$, which are inserted at the start of the transaction. After indexing all transactions, a block $B$ is created including the block index $B_{i}$, block header $B_{h}$, previous block header $B_{h}^{-}$, nonce $B_{n}$, timestamp $B_{t}, M_{r}, C_{T}$, block miner $B_{m}\left(Q_{p}\right)$, and block size $B_{s}$. For broadcasting the block to the consortium network, the authority must sign it with $k_{p}$ and provide the signature $S_{A}$ so that other authorities can authenticate the block by verifying $S_{A}$ with $B_{m}$.

An elliptic curve Deffie-Hellman (ECDH) key exchangebased mutual authentication protocol for the authorities of the network is proposed. Let $k_{A}$ and $k_{B}$ be the private keys and $Q_{A}=k_{A} G$ and $Q_{B}=k_{B} G$ be the public keys of authorities A and $B$, respectively. The mutual authentication scenario of the two authorities is illustrated in Fig. 5, and the authentication steps are described as follows:

1) Authority A sends a join request Join_Req $\left(Q_{A}\right)$ to authority B.

2) Authority B checks whether the request is valid or not by confirming that the received public key is registered in the network. If $Q_{A}$ is found to be registered in the network, authority B sends a join response Join_Res $\left(Q_{B}\right)$ to authority $\mathrm{A}$.

3) Upon receiving the join response, authority A computes $\phi=k_{A} Q_{B}$ and sends an accept request Accept_Req $(\phi)$ to authority $\mathrm{B}$.

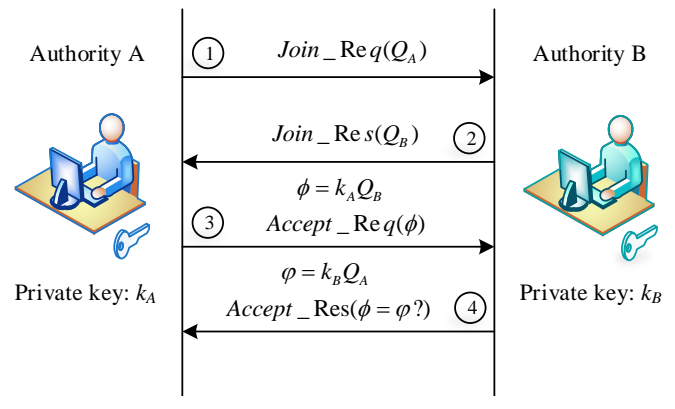

Fig. 5. Mutual authentication scheme for proposed consortium network.

4) Authority B performs $\varphi=k_{B} Q_{A}$ and checks whether $\phi=\varphi$. If the condition $\phi=\varphi$ is satisfied, a secure channel is established between them. An accept response $\operatorname{Accept} t_{-} \operatorname{Res}(\phi \stackrel{?}{=} \varphi)$ is sent to authority A. If $\phi$ does not match $\varphi$, the communication is terminated.

\section{Security Analyses}

A. Lemma 1: Retrieving private key by inverse point multiplication $\left[k=Q P^{-1}\right]$

Retrieving $k$ from the points $P$ and $Q$ by performing inverse point multiplication, such as $k=Q P^{-1}$, is known as the elliptic curve discrete logarithm problem (ECDLP). It is theoretically possible but practically difficult to solve the ECDLP over a large prime field. For example, ECDLPs over 109- and 113-bit $\mathbb{F}_{p}$ take 549 and 1045 days to be solved by employing 10,000 workstations together on the Internet, respectively [17], [21]. Hence, solving an ECDLP over 256-bit $\mathbb{F}_{p}$ is clearly almost impossible.

\section{B. Lemma 2: Proof of authentication $[\phi=\varphi]$}

Let $\left(k_{A}, Q_{A}\right)$ and $\left(k_{B}, Q_{B}\right)$ be the private-public key pairs of authorities $\mathrm{A}$ and $\mathrm{B}$, respectively, where

$$
\begin{aligned}
& Q_{A}=k_{A} G \\
& Q_{B}=k_{B} G
\end{aligned}
$$

When authority A attempts to connect with authority B, two $\mathrm{ECDH}$ keys are generated separately for mutual authentication such that

$$
\begin{aligned}
& \phi=k_{A} Q_{B}=k_{A}\left(k_{B} G\right)=k_{A} k_{B} G \\
& \varphi=k_{B} Q_{B}=k_{B}\left(k_{A} G\right)=k_{A} k_{B} G
\end{aligned}
$$

If $\phi$ does not match $\varphi$, authority A is not the owner of $Q_{A}$ because the authority does not have the private key $k_{A}$. In such a case, the authentication fails.

\section{Lemma 4: Proof of content creation}

During the registration process, a CID is broadcast to the consortium network rather than revealing the content itself. The CID is a public key $Q_{c}$ produced by converting the content into a private key integer $k_{c}$. Only the content owner can prove ownership of the CID by signing a transaction using 
the secondary private key $k_{s}$, which is the combination of the owner's primary private key $k_{p}$ and $k_{c}$ :

$$
k_{s}=k_{p}+k_{c}
$$

The corresponding secondary public key $Q_{s}$ can be computed by performing ECPM with $k_{s}$ as follows:

$$
Q_{s}=k_{s} G
$$

According to the properties of an elliptic curve,

$$
\begin{aligned}
& k_{s} G=\left(k_{p}+k_{c}\right) G \\
& k_{s} G=k_{p} G+k_{c} G \\
& Q_{s}=Q_{p}+Q_{c}
\end{aligned}
$$

The registering transaction is signed using $k_{s}$, and the signature is verified with $Q_{s}$. By combining $k_{p}$ and $k_{c}$, a relation between the owner's wallet and the unregistered content is established. In the case of a false owner, it is mathematically possible to generate a duplicate secondary public key $Q_{s}^{f}$ by simply adding the CID $Q_{c}$ to the primary public key of the false owner $Q_{p}^{f}$, but the corresponding secondary private key $k_{s}^{f}$ required to sign the transaction cannot be generated without knowing $k_{c}$. This is because a public key can be computed from a private key but the reverse operation is not possible due to the ECDLP. Hence, the false owner cannot supply a valid signature with their false transaction, and thus the transaction is refused by the authorities of the network.

The content creator is advised not to reveal the content as well as $k_{c}$ before the transaction being confirmed and recorded in the blockchain.

\section{Lemma 5: Proof of ownership transfer}

Say a copyright buyer $\left\{k_{p_{b}}, Q_{p_{b}}\right\}$ wants to buy a copyright $\left\{T_{h}, Q_{c}, Q_{t_{s}}\right\}$ from a seller $\left\{k_{p_{s}}, Q_{p_{s}}, k_{t_{s}}\right\}$. To sell the copyright, the seller receives payment from the buyer and delivers the tertiary private key $k_{t_{s}}$. Upon receiving $k_{t_{s}}$ from the seller, the buyer generates a secondary private-public key pair $\left(k_{s_{b}}, Q_{s_{b}}\right)$ as follows:

$$
\begin{aligned}
& k_{s_{b}}=k_{p_{b}}+k_{t_{s}} \\
& Q_{s_{b}}=k_{s_{b}} G
\end{aligned}
$$

According to the properties of an elliptic curve,

$$
\begin{aligned}
& k_{s_{b}} G=\left(k_{p_{b}}+k_{t_{s}}\right) G \\
& k_{s_{b}} G=k_{p_{b}} G+k_{t_{s}} G \\
& Q_{s_{b}}=Q_{p_{b}}+Q_{t_{s}}
\end{aligned}
$$

As in the registering transaction, the trading transaction is signed using $k_{s_{b}}$, which can be verified with $Q_{s_{b}}$. A false buyer $\left\{k_{p_{b}}^{f}, Q_{p_{b}}^{f}\right\}$ can computes $Q_{s_{b}}^{f}$ by simply adding $Q_{t_{s}}$ to $Q_{p_{b}}^{f}$, but they cannot provide a valid signature without having $k_{t_{s}}$.

\section{Implementation Results \& Performance ANALYSES}

The proposed system was implemented in Python with five virtual machines on the Oracle VM VirtualBox 6.1 installed in a host computer (Processor: Intel Core i5-10400 CPU@ 2.9 GHz, Memory: 48 GB): VM0 (Memory: 4 GB, Core: 1), VM1 (Memory: $16 \mathrm{~GB}$, Cores: 4), VM2 (Memory: $12 \mathrm{~GB}$, Cores: 3), VM3 (Memory: $8 \mathrm{~GB}$, Cores: 2), and VM4 (Memory: 4 GB, Cores: 1), each with different Internet protocol (IP) addresses. The four nodes VM1, VM2, VM3, and VM4 were considered as four authorities, here referred to as authorities A, B, C, and $\mathrm{D}$, respectively. A full node for the copyright federation, which provides tokens and holds a copy of the complete blockchain, was installed in VM0. A light node for a user who makes transactions without downloading the blockchain was also installed there. The user continuously conducted transactions and broadcast them to the consortium network. The user datagram protocol (UDP) was used for broadcasting transactions and blocks. Since the UDP supports only $64 \mathrm{kB}$ of data transfer in a single packet, the maximum capacity (block volume) of the system is 70 single-authored transactions per block, which will occupy approximately $63 \mathrm{kB}$ of memory. The block size grows for multi-authored transactions depending on the number of joint authors. However, the transmission capacity and number of transactions per block can be increased by adopting a packet-slicing approach, which we avoid for now in our small-scale experiment.

A round-robin schedule was set as $\mathrm{A} \rightarrow \mathrm{B} \rightarrow \mathrm{C} \rightarrow \mathrm{D} \rightarrow \mathrm{A}$ for selecting the step leaders of the PoA consensus. The sensitivity of the system was tested by performing experiments with separately varying the step duration or block volume, while fixing the other. The block volume was set to the maximum limit (70 Tx/block) and the step duration $\delta$ was varied from 5 to $8 \mathrm{~s}$. The corresponding mining results were recorded to analyze the system performance and determine the optimum value of $\delta$. Table I lists the block mining results of the first three rounds for different values of $\delta$. According to PoA, a block must receive acceptance from the majority of authorities to be added to the blockchain. Hence, for four authorities, the proposed block at a given step is added to the blockchain as soon as it receives acceptance from any two non-leader authorities of that step. At each step, the step leader creates a block with valid transactions (if available), broadcasts the block to the network, and receives at least two acceptances of the block. All these tasks should be completed within time period $\delta$. If the total latency exceeds $\delta$, the block is yet accepted unless it faces the next block. However, if a step leader does not receive sufficient acceptances of the previous step leader's block before starting to create a new block, two consecutive blocks will contain the same block header and common transactions. In such a case, the former is accepted if it receives sufficient acceptances before the appearance of the latter block, and the latter one is rejected when it is proposed. Rejection of a block or missing a turn means increasing the block time by an interval of $\delta$, which is unexpected. Setting the step duration to $\delta$ means the network expects to add new blocks to the blockchain after each $\delta$ time interval. Therefore, the value of $\delta$ should be sufficient for a block to be proposed by the current step leader and added by the next step leader to the blockchain with sufficient acceptances within this time frame. As shown in the table, all the 16 blocks were successfully added to the blockchain in the case of $\delta=7 \mathrm{~s}$ 
Table I

Mining Results of The First Three Rounds for Different Step Durations And The Maximum Block Volume

\begin{tabular}{|c|c|c|c|c|c|c|c|c|c|c|c|c|c|}
\hline \multirow{3}{*}{$\begin{array}{l}\text { Step } \\
\text { index }\end{array}$} & \multirow{3}{*}{$\begin{array}{l}\text { Step } \\
\text { leader }\end{array}$} & \multicolumn{6}{|c|}{$\delta=7 \mathrm{~s}$} & \multicolumn{6}{|c|}{$\delta=5 \mathrm{~s}$} \\
\hline & & \multirow{2}{*}{$\begin{array}{c}\text { Block proposal } \\
\text { time (s) }\end{array}$} & \multicolumn{4}{|c|}{ Block confirmation time (s) } & \multirow{2}{*}{$\begin{array}{l}\text { Mining } \\
\text { result }\end{array}$} & \multirow{2}{*}{$\begin{array}{l}\text { Block proposal } \\
\text { time (s) }\end{array}$} & \multicolumn{4}{|c|}{ Block confirmation time (s) } & \multirow{2}{*}{$\begin{array}{c}\text { Mining } \\
\text { result }\end{array}$} \\
\hline & & & A & $\mathrm{B}$ & $\mathrm{C}$ & $\mathrm{D}$ & & & A & $\mathrm{B}$ & $\mathrm{C}$ & $\mathrm{D}$ & \\
\hline 0 & A & 1.47 & 3.12 & 3.75 & 3.53 & 3.14 & $\checkmark$ & 1.49 & 3.31 & 3.26 & 3.17 & 3.70 & $\checkmark$ \\
\hline 1 & $\mathrm{~B}$ & 1.45 & 3.23 & 3.34 & 3.36 & 3.44 & $\checkmark$ & 1.68 & 3.56 & 3.44 & 3.58 & 4.42 & $\checkmark$ \\
\hline 2 & $\mathrm{C}$ & 1.51 & 3.34 & 3.34 & 3.53 & 4.38 & $\checkmark$ & 1.59 & - & - & - & - & $x$ \\
\hline 3 & $\mathrm{D}$ & 1.60 & 3.50 & 3.52 & 3.45 & 3.25 & $\checkmark$ & 1.60 & 3.37 & 3.81 & 3.85 & 4.05 & $\checkmark$ \\
\hline 4 & A & 1.47 & 3.23 & 3.34 & 3.39 & 4.03 & $\checkmark$ & 1.54 & 3.70 & 3.53 & 3.44 & 3.98 & $\checkmark$ \\
\hline 5 & B & 1.53 & 3.30 & 3.31 & 3.47 & 4.06 & $\checkmark$ & 1.90 & - & - & - & - & $\times$ \\
\hline 6 & $\mathrm{C}$ & 1.50 & 3.22 & 3.38 & 3.42 & 3.48 & $\checkmark$ & 1.52 & 3.47 & 3.50 & 3.34 & 3.45 & $\checkmark$ \\
\hline 7 & $\mathrm{D}$ & 1.56 & 3.23 & 3.28 & 3.39 & 4.56 & $\checkmark$ & 1.83 & - & - & - & - & $x$ \\
\hline 8 & A & 1.52 & 3.42 & 3.28 & 3.52 & 3.47 & $\checkmark$ & 1.52 & 3.34 & 3.50 & 3.34 & 3.77 & $\checkmark$ \\
\hline 9 & B & 1.53 & 3.22 & 3.30 & 3.37 & 3.97 & $\checkmark$ & 2.03 & - & - & - & - & $x$ \\
\hline 10 & $\mathrm{C}$ & 1.47 & 3.33 & 3.33 & 3.61 & 4.59 & $\checkmark$ & 1.52 & 3.53 & 3.78 & 3.36 & 3.48 & $\checkmark$ \\
\hline 11 & $\mathrm{D}$ & 1.59 & 3.37 & 3.48 & 3.52 & 3.75 & $\checkmark$ & 1.91 & - & - & - & - & $\times$ \\
\hline
\end{tabular}

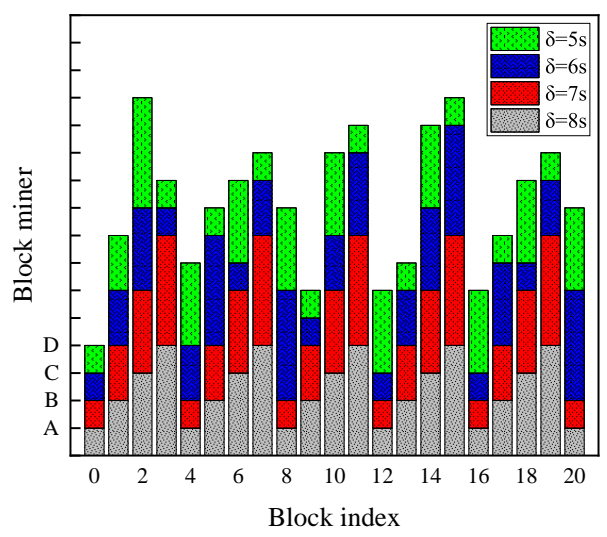

Fig. 6. Miners of the first 20 blocks for variable step duration.

because the step duration was sufficient for the step leaders to propose and accept the blocks. In the case of $\delta=5 \mathrm{~s}$, two step leaders failed in each round because they received the previous block exceeding the deadline. For example, the block time (proposal + confirmation) at step 1 with respect to authority $\mathrm{C}$ was $5.26 \mathrm{~s}$, which is greater than $\delta$. This delay affected the acceptance of the next block. The block proposed by $\mathrm{C}$ at step 2 was rejected because it contained the same block header as the block proposed at step 1. In this manner, one or more blocks were rejected in each round when the step duration was less than $7 \mathrm{~s}$. To overcome the problem of block rejection, the minimum step duration of this network can be set to $7 \mathrm{~s}$. However, this setting varies from network to network depending on the network performance, such as incoming transaction rate, block volume, computing power of authorities, response time of authorities, and network throughput.

Fig. 6 illustrates the miners of blocks 0-20 for different values of $\delta$. It can be seen that no authority missed a turn when $\delta$ was $7 \mathrm{~s}$ or $8 \mathrm{~s}$, whereas one and two turns were missed in each round for $\delta=6 \mathrm{~s}$ and $5 \mathrm{~s}$, respectively. As $\delta$ decreases, the probability of missing turns by some of the authorities increases, and vice versa. Fig. 7 shows the epoch times of the first 20 blocks for variable step duration. The

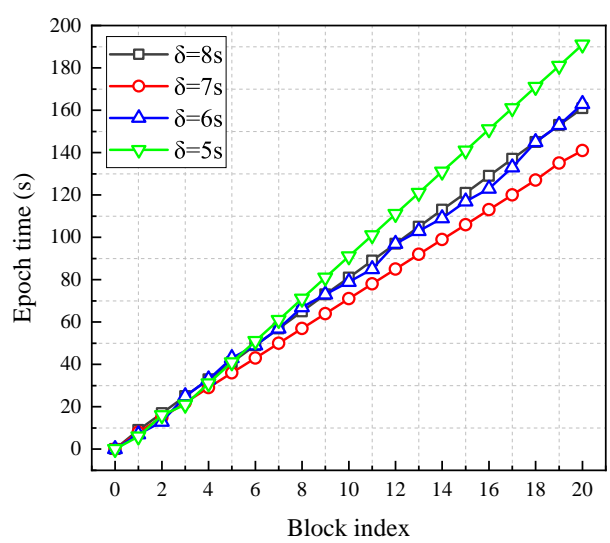

Fig. 7. Epoch times of the first 20 blocks for variable step duration.

epoch time of a particular block is the difference between the timestamps of that block and block 0 . It is observed that a step duration of $7 \mathrm{~s}$ takes the lowest time to add 20 blocks to the blockchain; hence, it is the optimum value of $\delta$ for this network. When some turns are missed in a round, it increases the total time required to mine a fixed number of blocks. Hence, step duration $5 \mathrm{~s}$ is the worst choice because many turns are missed in this case. Although no turn is missed when $\delta=8 \mathrm{~s}$, it takes $160 \mathrm{~s}$ to mine a total of 20 blocks, whereas the setting $\delta=7 \mathrm{~s}$ requires only $140 \mathrm{~s}$ to mine the same number of blocks.

Fig. 8(a) illustrates the block times of the first 20 blocks for variable step duration and 70 transactions per block. It can be seen that a step duration of $\geq 7 \mathrm{~s}$ achieves almost a constant block time, which means that the blocks are periodically mined. This meets the expectation of the network in terms of block interval. However, block time for a lower step duration appears to be an unstable curve because the blocks are not mined timely. The effect of reducing block size by decreasing the number of transactions per block for a fixed step duration is depicted in Fig. 8(b). The step duration was fixed to $5 \mathrm{~s}$, the block volume was varied as 70, 60, and 50 transactions per block, and the corresponding block time was measured. As the block volume decreased, the percentage of turn failure 


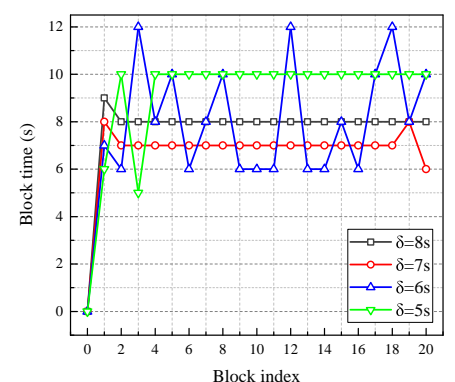

(a)

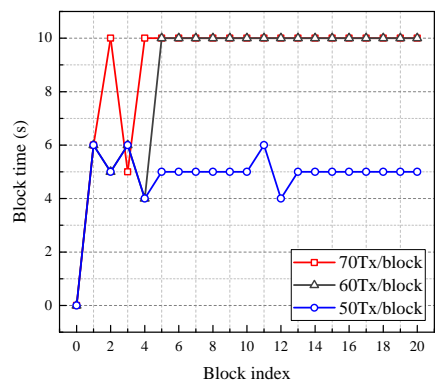

(b)
Fig. 8. Block times of the first 20 blocks for (a) variable step duration and fixed block volume (70 Tx/block) and (b) fixed step duration (5s) and variable block volume.

Table II

PERFormance COMParison OF The Authorities AND Mining SUMMARY

\begin{tabular}{|c|c|c|c|c|c|}
\hline \multicolumn{2}{|c|}{ Authority } & A & B & $\mathrm{C}$ & $\mathrm{D}$ \\
\hline \multicolumn{2}{|c|}{ ECPM time (ms) } & 3.6 & 3.6 & 3.6 & 3.6 \\
\hline \multicolumn{2}{|c|}{ ECDSA signing time $(\mathrm{ms})$} & 3.7 & 3.7 & 3.8 & 3.9 \\
\hline \multicolumn{2}{|c|}{ ECDSA verification time (ms) } & 7.3 & 7.4 & 7.4 & 7.6 \\
\hline \multicolumn{2}{|c|}{ Tx validation speed $(\mathrm{ms} / \mathrm{Tx})$} & 21.7 & 21.9 & 22.1 & 23.7 \\
\hline \multirow{3}{*}{$\begin{array}{l}\text { Block } \\
\text { preparation } \\
\text { time (s) }\end{array}$} & $70 \mathrm{Tx} / \mathrm{block}$ & 1.50 & 1.52 & 1.55 & 1.68 \\
\hline & $60 \mathrm{Tx} / \mathrm{block}$ & 1.30 & 1.31 & 1.32 & 1.40 \\
\hline & $50 \mathrm{Tx} / \mathrm{block}$ & 1.10 & 1.11 & 1.11 & 1.19 \\
\hline \multirow{3}{*}{$\begin{array}{l}\text { Average } \\
\text { response } \\
\text { time (s) }\end{array}$} & $70 \mathrm{Tx} /$ block & 2.95 & 3.23 & 3.25 & 3.56 \\
\hline & $60 \mathrm{Tx} /$ block & 2.78 & 2.80 & 2.82 & 2.96 \\
\hline & $50 \mathrm{Tx} / \mathrm{block}$ & 2.23 & 2.36 & 2.41 & 2.45 \\
\hline \multirow{2}{*}{$\begin{array}{c}\text { Throughput } \\
\text { (Kbps) }\end{array}$} & $\delta=7 \mathrm{~s}$ & 45.36 & 45.36 & 43.69 & 44.21 \\
\hline & $\delta=5 \mathrm{~s}$ & 45.36 & 42.90 & 43.25 & 32.63 \\
\hline \multirow{2}{*}{$\begin{array}{l}\text { Average network } \\
\text { throughput (Kbps) }\end{array}$} & $\delta=7 \mathrm{~s}$ & \multicolumn{4}{|c|}{264.84} \\
\hline & $\delta=5 \mathrm{~s}$ & \multicolumn{4}{|c|}{252.97} \\
\hline \multirow{2}{*}{$\begin{array}{l}\text { Maximum network } \\
\text { throughput (Mbps) }\end{array}$} & $\delta=7 \mathrm{~s}$ & \multicolumn{4}{|c|}{1.11} \\
\hline & $\delta=5 \mathrm{~s}$ & \multicolumn{4}{|c|}{1.35} \\
\hline \multirow{3}{*}{$\begin{array}{c}\text { Block } \\
\text { size }(\mathrm{kB})\end{array}$} & $70 \mathrm{Tx} / \mathrm{block}$ & \multicolumn{4}{|c|}{63} \\
\hline & $60 \mathrm{Tx} / \mathrm{block}$ & \multicolumn{4}{|c|}{54} \\
\hline & $50 \mathrm{Tx} /$ block & \multicolumn{4}{|c|}{45} \\
\hline \multicolumn{2}{|c|}{ Transaction size (bytes) } & \multicolumn{4}{|c|}{895} \\
\hline \multicolumn{2}{|c|}{ Transaction throughput (Tx/s) } & \multicolumn{4}{|c|}{10} \\
\hline \multicolumn{2}{|c|}{ Mutual authentication time (ms) } & \multicolumn{4}{|c|}{31.5} \\
\hline
\end{tabular}

and hence block time decreased. For 50 transactions per block, the average block time reached the step duration. Therefore, $5 \mathrm{~s}$ is the optimum step duration for a block volume of 50 transactions. In other words, this network can process 10 transactions per second, which is the transaction throughput of the network.

A performance comparison of the authorities and mining summary of the network are presented in Table II. The throughput of individual authorities was measured using the Wireshark 3.4.6 network traffic monitoring tool. Authority A holds the highest computing power and throughput because it has the best hardware configuration. The performance of the authorities decreases in order from A to D. Although B has higher processing speed than $\mathrm{C}$, the throughput of $\mathrm{B}$ is lower in the case of $\delta=5 \mathrm{~s}$ due to rejection of several block proposals. The average throughput for a step duration of $5 \mathrm{~s}$ is comparatively low, but the maximum throughput is high due to non-periodic block proposal and acceptance in this case.
For a better performance, it is preferred that the network be operated with a higher average throughput by periodic block mining.

\section{CONCLUSION}

A decentralized copyright system has been proposed in this paper for cross-border copyright protection based on a consortium blockchain with the PoA consensus algorithm. Unlike conventional copyright systems, the proposed system does not require any centralized server to store copyrighted content. Instead, blockchain is used to store the metadata of copyrighted content. This creates a synchronized platform for copyright investigation of worldwide digital content. It also eliminates the need for an encryption-decryption process in copyright management. Copyright is provided to registered users only so that punishment can be applied to copyright infringements. Interested people can easily inquire about content to know whether it is copyrighted or not. Thus, they can become conscious of copyrighted works and will not unknowingly violate copyright laws. Based on the overall performance analyses, it can be concluded that the system can be implemented on a large scale and commercialized for international copyright management.

\section{FUTURE WORK}

The current form of our proposed system supports copyright registration of individual works belonging to a single or multiple authors and the transfer of the entire ownership to a new owner (assignment). As future work, we plan to enable copyrighting of a collective work that assembles a number of separate works into one larger work (e.g., a newspaper or scholarly journal). We also plan to add the feature of licensing specific rights on a copyrighted work.

\section{ACKNOWLEDGMENT}

This work was supported by the National Research Foundation (NRF) of Korea grant funded by the Ministry of Science and ICT (MSIT) of the Korean government, under Grant No. NRF-2021R1A2C2012476. The authors would like to thank Mpyana Mwamba Merlec ( $\mathrm{PhD}$ candidate, IBE Lab, Korea University) for his support in creating the experimental environment.

\section{REFERENCES}

[1] M. Trimble, "Undetected conflict-of-laws problems in cross-border online copyright infringement cases," N. C. J. L. \& Tech., vol. 18, no. 1, pp. 119-159, 2016.

[2] Summaries of conventions, treaties and agreements administered by WIPO, Geneva, Switzerland: WIPO, 2013, pp. 1-60.

[3] Berne convention for the protection of literary and artistic works, Geneva, Switzerland: WIPO, Sep. 1979, pp. 1-59.

[4] E. Fiordalisi, "The tangled web: Cross-border conflicts of copyright law in the age of Internet sharing," Loy. U. Chi. Int'l L. Rev., vol. 12, no. 2, pp. 197-213, 2015.

[5] Compendium of U.S. copyright office practices, 3rd ed. Washington, DC: U.S. Copyright Office, Dec. 2014, pp. 1-1301.

[6] A. Savelyev, "Copyright in the blockchain era: Promises and challenges," Comput. Law Secur. Rev., vol. 34, no. 3, pp. 550-561, June 2018.

[7] J. Benet, "IPFS-content addressed, versioned, P2P file system," arXiv preprint, July 2014. [Online]. Available: https://arxiv.org/abs/1407.3561. 
[8] W. Entriken, D. Shirley, J. Evans, and N. Sachs, "EIP-721: ERC-721 nonfungible token standard," Ethereum Improvement Proposals, Jan. 2018. [Online]. Available: https://eips.ethereum.org/EIPS/eip-721.

[9] A. Kumar, S. P. Ghrera, and V. Tyagi, "An ID-based secure and flexible buyer-seller watermarking protocol for copyright protection," Pertanika J. Sci. \& Technol., vol. 25, no. 1, pp. 57-76, Jan. 2017.

[10] S. H. Low, N. F. Maxemchuk, and A. M. Lapone, "Document identification for copyright protection using centroid detection," IEEE Trans. Commun., vol. 46, pp. 372-383, Mar. 1998.

[11] M. D. Swanson, M. Kobayashi, and A. H. Tewfik, "Multimedia dataembedding and watermarking technologies," Proc. IEEE, vol. 86, pp. 1064-1087, June 1998

[12] J. Hernandez and F. Perez-Gonzalez, "Statistical analysis of watermarking schemes for copyright protection," Proc. IEEE, vol. 87, pp. 11421166, July 1999.

[13] G. Voyatzis and I. Pitas, "The use of watermarks in the protection of digital multimedia products," Proc. IEEE, vol. 87, pp. 1197-1207, July 1999.

[14] W.-N. Lie, G.-S. Lin, and S.-L. Cheng, "Dual protection of JPEG images based on informed embedding and two-stage watermark extraction," IEEE Trans. Inf. Forensics Security, vol. 1, no. 3, pp. 330-341, Sep. 2006.

[15] J. Tzeng, W.-L. Hwang, and I.-L. Chern, "An asymmetric subspace watermarking method for copyright protection," IEEE Trans. Signal Process., vol. 53, no. 2, pp. 784-792, Feb. 2005.

[16] N. A. Memon and A. Alzahrani, "Prediction-based reversible watermarking of CT scan images for content authentication and copyright protection," IEEE Access, vol. 8, pp. 75448- 75462, Apr. 2020.

[17] Y. Zhang, C. Wang, X. Wang, and M. Wang, "Feature-based image watermarking algorithm using SVD and APBT for copyright protection," Future Internet, vol. 9, no. 2, p. 13, Apr. 2017.

[18] S. Zafeiriou, A. Tefas, and I. Pitas, "Blind robust watermarking schemes for copyright protection of 3D mesh objects," IEEE Trans. Vis. Comput. Graphics, vol. 11, no. 5, pp. 596-607, Oct. 2005.

[19] X.-L. Liu, C.-C. Lin, and S.-M. Yuan, "Blind dual watermarking for color images' authentication and copyright protection," IEEE Trans. Circuits Syst. Video Technol., vol. 28, no. 5, pp. 1047-1055, May 2016.

[20] Q. Su, Y. Niu, H. Zou, Y. Zhao, and T. Yao, "A blind double color image watermarking algorithm based on QR decomposition," Multimedia Tools Appl., vol. 72, no. 1, pp. 987-1009, Sep. 2014.

[21] T.-H. Chen, G. Horng, and W.-B. Lee, "A publicly verifiable copyrightproving scheme resistant to malicious attacks," IEEE Trans. Ind. Electron., vol. 52, no. 1, pp. 327-334, Feb. 2005.

[22] Guang, F. Xiaoping, J. Wangdong, L. Fenghua, and J. Yuewei, "Obfuscation-based watermarking for mobile service application copyright protection in the cloud," IEEE Access, vol. 7, pp. 38162-38167, Jan. 2019.

[23] F. Ernawan and M. N. Kabir, "An improved watermarking technique for copyright protection based on tchebichef moments," IEEE Access, vol. 7, pp. 151985-152003, Oct. 2019.

[24] M. T. Ahvanooey, Q. Li, H. J. Shim, and Y. Huang, "A comparative analysis of information hiding techniques for copyright protection of text documents," Secur. Commun. Netw., vol. 2018, Apr. 2018, Art. no. 5325040 .

[25] J. T. Brassil and N. F. Maxemchuk, "Copyright protection for the electronic distribution of text documents," Proc. IEEE, vol. 87, no. 7 , pp. 1181-1196, July 1999.

[26] U. Khadam et al., "Digital watermarking technique for text document protection using data mining analysis," IEEE Access, vol. 7, pp. 64955-64965, May 2019.

[27] Y. Shi, H. Yang, M. Gong, X. Liu, and Y. Xia, "A fast and robust key frame extraction method for video copyright protection," J. Electr. Comput. Eng., vol. 2017, Mar. 2017, Art. no. 1231794.

[28] X. Yu, C. Wang, and X. Zhou, "A survey on robust video watermarking algorithms for copyright protection," Appl. Sci., vol. 8, no. 10, p. 1891, Oct. 2018.

[29] A. A. Elrowayati, M. A. Alrshah, M. F. L. Abdullah, and R. Latip, "HEVC watermarking techniques for authentication and copyright applications: Challenges and opportunities," IEEE Access, vol. 8, pp. 114172 114189, June 2020

[30] J.-U. Hou, D. Kim, W.-H. Ahn, and H.-K. Lee, "Copyright protections of digital content in the age of 3D printer: Emerging issues and survey," IEEE Access, vol. 6, pp. 44082-44093, Aug. 2018.

[31] M. Holland, C. Nigischer, and J. Stjepandic, "Copyright protection in additive manufacturing with blockchain approach," Adv. Transdisciplinary Eng., vol. 5, pp. 914-921, July 2017.
[32] L. Xiao, W. Huang, Y. Xie, W. Xiao, and K.-C. Li, "A blockchainbased traceable IP copyright protection algorithm," IEEE Access, vol. 8, pp. 49532-49542, Jan. 2020.

[33] G. Heo, D. Yang, I. Doh, and K. Chae, "Efficient and secure blockchain system for digital content trading," IEEE Access, vol. 9, pp. 77438-77450, May 2021.

[34] Understanding copyright and related rights, 2nd ed. Geneva, Switzerland: WIPO, 2016, pp. 1-40.

[35] W. Yao, J. Ye,R. Murimi, and G. Wang, "A survey on consortium blockchain consensus mechanisms," arXiv preprint, Feb. 2021. [Online]. Available: https://arxiv.org/abs/2102.12058.

[36] S. De Angelis, L. Aniello, R. Baldoni, F. Lombardi, A. Margheri, and V. Sassone, "PBFT vs proof-of-authority: Applying the CAP theorem to permissioned blockchain," in Proc. Italian Conf. Cyber Secur., Milan, Italy, June 2018, pp. 1-11.

[37] Y. Sompolinsky and A. Zohar, "Secure high-rate transaction processing in bitcoin," in Proc. Int. Conf. Financial Cryptogr. Data Secur., San Juan, Puerto Rico: Springer, 2015, pp. 507-527.

[38] L. Wang, X. Shen, J. Li, J. Shao, and Y. Yang, "Cryptographic primitives in blockchains," J. Netw. Comput. Appl., vol. 127, pp. 43-58, Feb. 2019.

[39] D. Hankerson, A. Menezes, and S. Vanstone, Guide to Elliptic Curve Cryptography. NY, USA: Springer-Verlag, 2004.

[40] M. Qu, "SEC 2: Recommended elliptic curve domain parameters," Certicom Res., Mississauga, ON, Canada, Tech. Rep. SEC2-Ver-0.6, 1999.

[41] M. M. Islam, M. S. Hossain, M. K. Hasan, M. Shahjalal, and Y. M. Jang, "FPGA implementation of high-speed area-efficient processor for elliptic curve point multiplication over prime field," IEEE Access, vol. 7, pp. 178811-178826, Dec. 2019.

\section{APPENDIX}

The prototype of our proposed system is available on Github: https://github.com/Mainul333/Decentralized-Global-Copyright-

System-Based-on-Consortium-Blockchain.git.

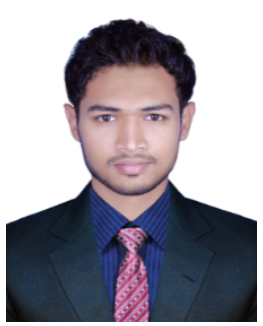

Md. Mainul Islam (Graduate Student Member, IEEE) received his BSc degree in Electrical and Electronic Engineering from Khulna University of Engineering \& Technology, Khulna, Bangladesh, in April 2018. He obtained his MSc degree in Electronics Engineering from Kookmin University, South Korea, in February 2021, receiving the Academic Excellence Award. Since March 2021, he has been working as a full-time Researcher in the Intelligent Blockchain Engineering Laboratory (IBEL) under the Department of Computer Science at Korea University, South Korea. His research interests include blockchain, smart contracts, cryptography, and data security.

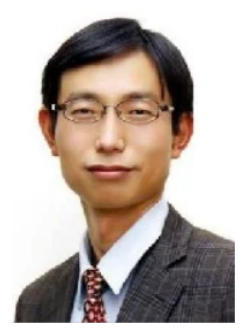

Hoh Peter IN (Member, IEEE) received his BSc degree in Computer Engineering from Korea University, South Korea, in 1990. He received his MSc degree in Computer Science from Korea University in 1992. He pursued his $\mathrm{PhD}$ degree in Computer Engineering from the University of Southern California (USC), USA, in 1998. In 1999, he became an assistant professor at Texas A\&M University, USA. In 2003, he joined the Department of Computer Science at Korea University as an assistant professor, where he is currently a professor. He is a chairperson of the Korea Society of Blockchain and the director of the Blockchain Research Institute, South Korea. His main research interests are blockchain, smart contract security, and software engineering. He received the ICRE 10 Year Most Influential Paper Award 2006. He has published over 120 research papers. 